\begin{tabular}{|c|c|}
\hline Title & $\begin{array}{l}\text { Developing wireless measurement system for building deployed } \\
\text { capacitive sensors with optimized RF front end circuit }\end{array}$ \\
\hline Authors & Jafer, Essa;O'Flynn, Brendan;Ó Mathúna, S. Cian;Buckley, John \\
\hline Publication date & 2008 \\
\hline Original Citation & $\begin{array}{l}\text { Jafer, E., O'Flynn, B., Ó Mathúna, S.C., Buckley, J. } 2008 . \\
\text { Developing wireless measurement system for building deployed } \\
\text { capacitive sensors with optimized RF front end circuit. In: IEEE, } \\
\text { Seventh IEEE Conference on Sensors: IEEE Sensors 2008. Lecce, } \\
\text { Italy 26-29 October } 2008 .\end{array}$ \\
\hline Type of publication & Conference item \\
\hline $\begin{array}{l}\text { Link to publisher's } \\
\text { version }\end{array}$ & $\begin{array}{l}\text { http://ieeexplore.ieee.org/xpl/tocresult.jsp? } \\
\text { isnumber=4716361\&isYear=2008 - 10.1109/ICSENS.2008.4716663 }\end{array}$ \\
\hline Rights & $\begin{array}{l}\text { (c) } 2008 \text { IEEE. Personal use of this material is permitted. However, } \\
\text { permission to reprint/republish this material for advertising } \\
\text { or promotional purposes or for creating new collective works } \\
\text { for resale or redistribution to servers or lists, or to reuse any } \\
\text { copyrighted component of this work in other works must be } \\
\text { obtained from IEEE. }\end{array}$ \\
\hline Download date & 2023-04-26 10:13:29 \\
\hline $\begin{array}{l}\text { Item downloaded } \\
\text { from }\end{array}$ & http://hdl.handle.net/10468/98 \\
\hline
\end{tabular}

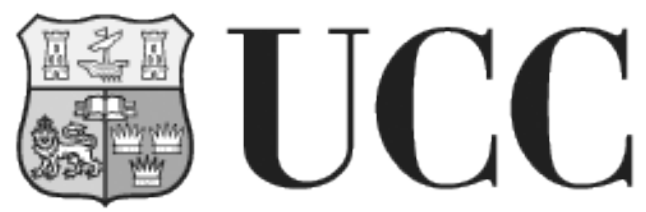

University College Cork, Ireland Coláiste na hOllscoile Corcaigh 


\title{
Developing Wireless Measurement System for Building Deployed Capacitive Sensors with Optimized RF Front End Circuit
}

\author{
Essa Jafer, Brendan O'Flynn, Cian O’Mathuna, and John Buckley \\ Tyndall National Institute, Lee Maltings, Prospect Row \\ Cork, Ireland \\ Essa.jafer@tyndall.ie
}

\begin{abstract}
- in this paper, a prototype of miniaturized, low power, bi-directional wireless sensor node for wireless sensor networks (WSN) was designed for doors and windows building monitoring. The capacitive pressure sensors have been developed particularly for such application, where packaging size and minimization of the power requirements of the sensors are the major drivers. The capacitive pressure sensors have been fabricated using a $2.4 \mu \mathrm{m}$ thick strain compensated heavily boron doped $\mathrm{SiGeB}$ diaphragm is presented. In order to integrate the sensors with the wireless module, the sensor dice was wire bonded onto TO package using chip on board (COB) technology. The telemetric link and its capabilities to send information for longer range have been significantly improved using a new design and optimization process. The simulation tool employed for this work was the Designer ${ }^{\circledR}$ tool from Ansoft Corporation.
\end{abstract}

\section{INTRODUCTION}

The issue of building efficient sensor nodes for wireless sensor network (WSN) involves several challenges as these designs include not only a union of the analog and digital circuit domains, but also the magnetic, mechanical, biological, chemical, or electrical domains [1]. From a communication point of view it is important to transmit data reliably while using the minimum amount of transceiver power. Also, communication range has to be maximized in order to reduce the number of nodes required and therefore the system cost. In the Building telemetry, there is always a need to develop remote systems that consume a low power and are compatible with a wide range of different possible deployed capacitive and resistive transducers types [2]. The importance of such system capable of monitoring various physical variables in the building environment, in order to improve domestic comfort, security and the rational use of energy, is rapidly increasing [3,4]. Due to this, we developed a miniaturized wireless node prototype for pressure monitoring that has a good operation life time, efficient transmission range and suitable for the range of pressure change.
The paper is organised as follows: In section two, the fabrication of the capacitive pressure sensors is explained. An overview description of the developed system is given in section three. In section four, some experimental results from testing the sensors are presented.

\section{SiGEB PRESSURE SENSOR}

The process relies on the silicon fusion bonding of two silicon wafers to seal the pressure sensor cavity and construct the device [6]. Strain compensated $\mathrm{Si}_{1-\mathrm{x}-\mathrm{y}} \mathrm{Ge}_{\mathrm{x}} \mathrm{B}_{\mathrm{y}}$ layers have been shown to exhibit excellent characteristics similar to those exhibited by simple heavily boron doped layers when used as etch stops in wet chemical etching solutions [6]. In this work, our sensors have been developed based on the advantage of these properties.

A schematic view of the developed sensor is depicted in Figure 1. The device consists of a cavity etched in a thick wet oxide, a fixed electrode and a flexible electrode. When pressure is applied, the flexible electrode deflects towards the fixed electrode and the device capacitance changes. In capacitive type pressure sensors it is this change that is of interest. Rather in pressure switches the value of pressure at which the flexible electrode will touch the fixed electrode is the important parameter.

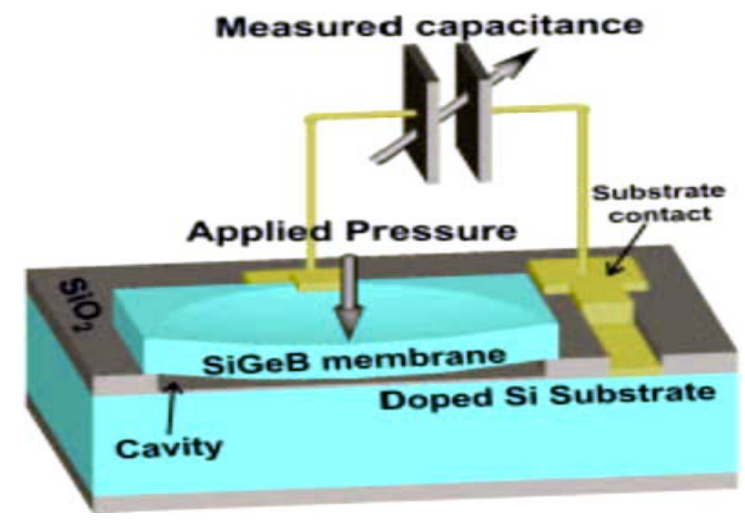

Figure 1. Schematic view of the pressure sensor 
In order to integrate the sensors with the wireless module, the sensor dice was wire bonded onto TO package using chip on board technology. A photograph of the packaged sensor is shown in Figure 2. Table.1 summarizes the main technical details of the pressure sensors

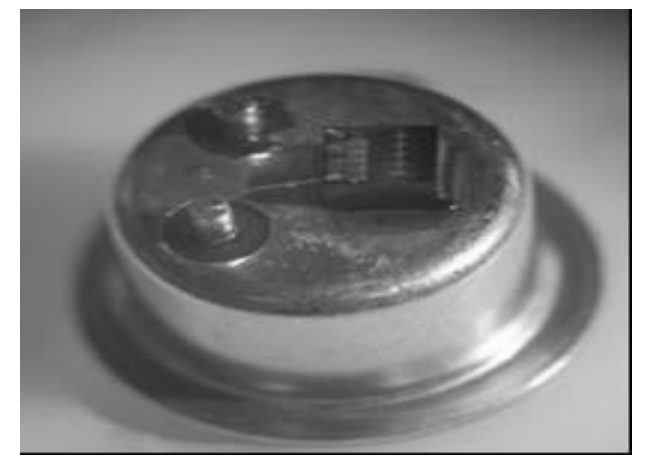

Figure 2. Schematic view of the pressure sensor

TABLE I. PRESSURE SENSORS SPECIFICATIONS

\begin{tabular}{|c|c|}
\hline Silicon die area & $1.1 \times 1.0 \mathrm{~mm}$ \\
\hline Baseline capacitance: & $4 \mathrm{pF}$ \\
\hline Resolution: & $1 \mathrm{fF} / \mathrm{mbar}$ \\
\hline Pressure range: & $0-100 \mathrm{mbar}$ \\
\hline
\end{tabular}

\section{WIRELESS SYSTEM OVERVIEW}

The whole system is made up of two main parts: the miniature RF transceiver and control base station, as illustrated in Figure 3. The base station sends commands to switch on the radio and configure the capacitive unit, and receives data packets sent by the miniaturized module. The system is half duplex, so data cannot be transmitted and received at the same time.

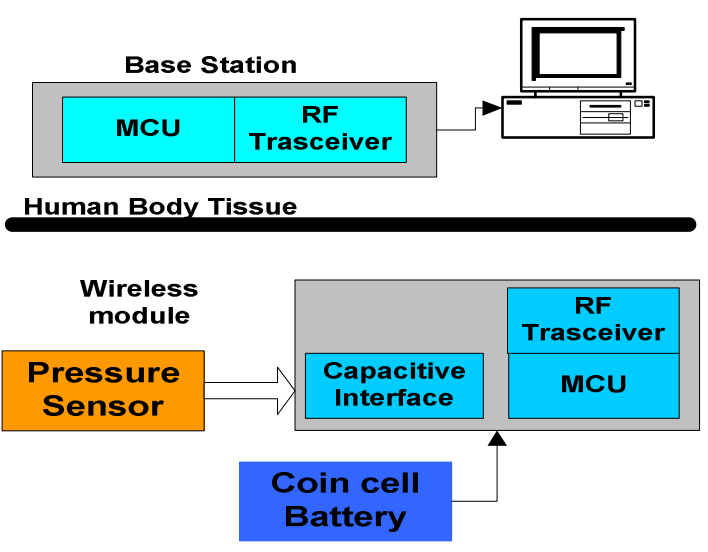

Figure 3. Block diagram of the developed system
The total size of the system printed circuit board (PCB) is $22.46 \times 20.168 \mathrm{~mm}$. The RF carrier frequency is in the 433 MHz ISM frequency band. Gaussian Frequency Shift Keying (GFSK) modulation has been adopted in the design with a data rate of $100 \mathrm{Kbps}$ and frequency deviation $\pm 50 \mathrm{KHz}$. This modulation type results in a more bandwidth effective transmission-link compared with ordinary FSK modulation. The data is internally Manchester encoded and decoded. That is, the effective symbol-rate of the link is 50kbps. By using internally Manchester encoding, no scrambling in the MCU is needed.

A 3 V Lithium coin cell battery of type CR2032 has been used for powering the miniaturized module. In order to investigate the battery lifetime, the supplied voltage of the battery has been measured for different power transmission values as shown in Figure 4.

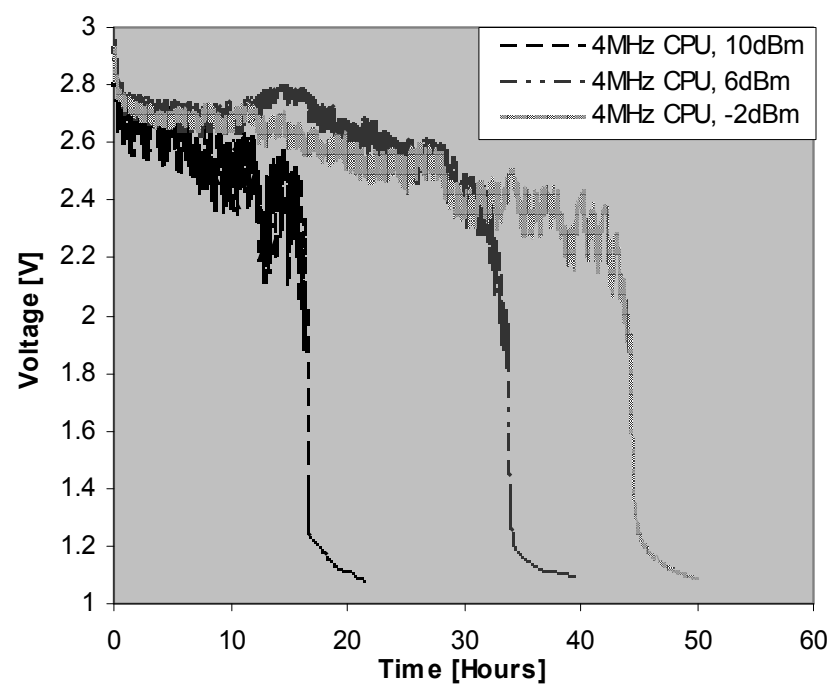

Figure 4. Result of operation time for the pressure module.

\section{A. Matching Network Optimization}

A few LC components have been selected to design the impedance matching network which does more than the matching task but also makes sure that harmonic and spurious emissions are kept low and that output power is not decreasing. This section presents the design and optimization processes used for the matching circuit. Figure 5 shows the design flow used during analysis. The design procedure starts with the design of the matching PCB layout, while following the manufactures guidelines closely. Following that, an electrical model of the matching subsection is developed in order to accurately predict the RF performance of the network. The simulation tool employed for this work was the Designer ${ }^{\circledR}$ tool from Ansoft Corporation [5, 6]. Ansoft Designer allows the design, optimization, and validation of component, circuit, and system performance. It includes a planar electromagnetic solver and a complete HF linear circuit simulator to model transmission lines and components. For initial simulations, the HF linear circuit simulator is used to develop an electrical model of the 
matching circuit as shown in Figure 6. Later on in the design cycle, more accurate simulations are possible by importing the finished matching layout directly from the PCB layout tool and specifying the s-parameter vendor models for the components. Once and accurate model has been developed, the values of the discrete components are optimized automatically in simulation.

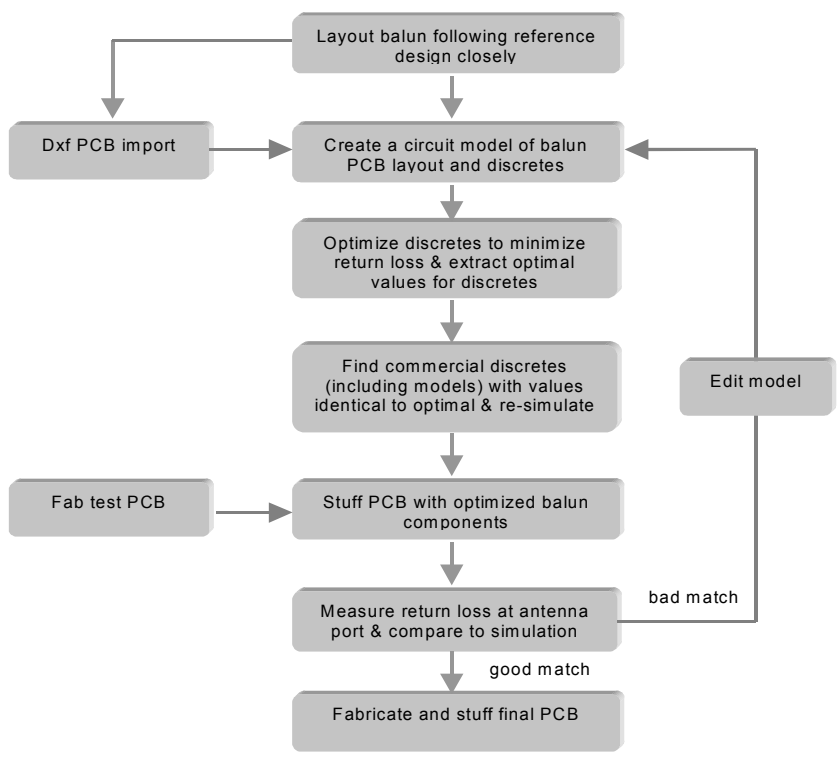

Figure 5. Optimization flow

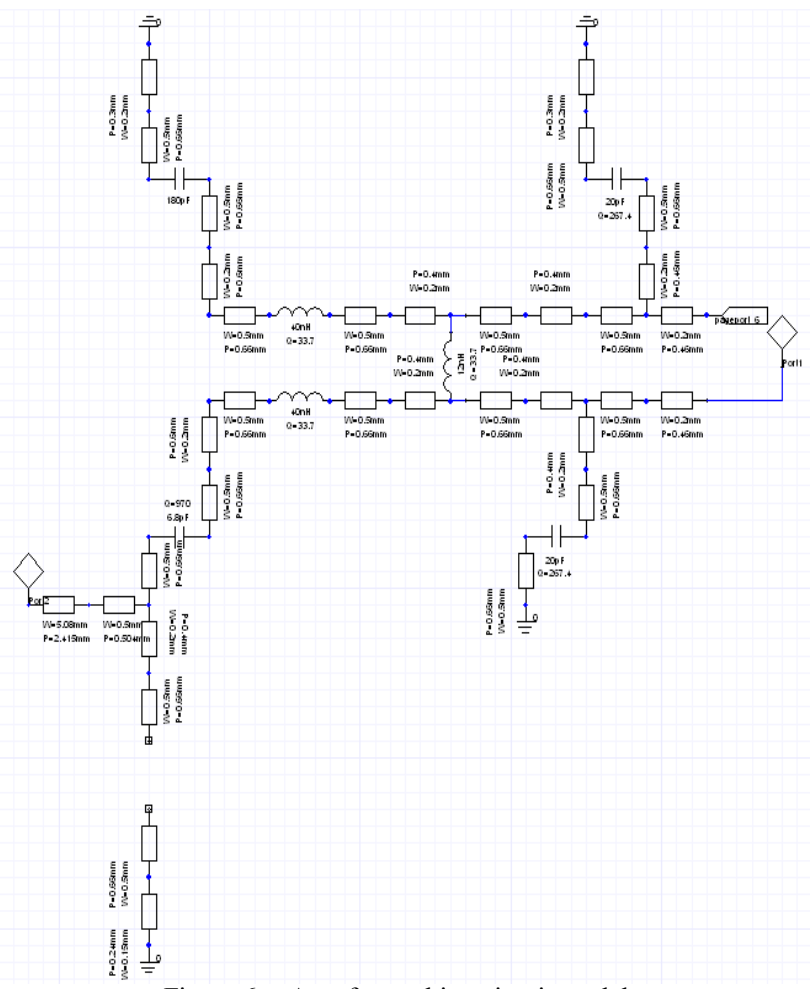

Figure 6. Ansoft matching circuit model
The planer model of the developed matching circuit model is shown in Figure 7.

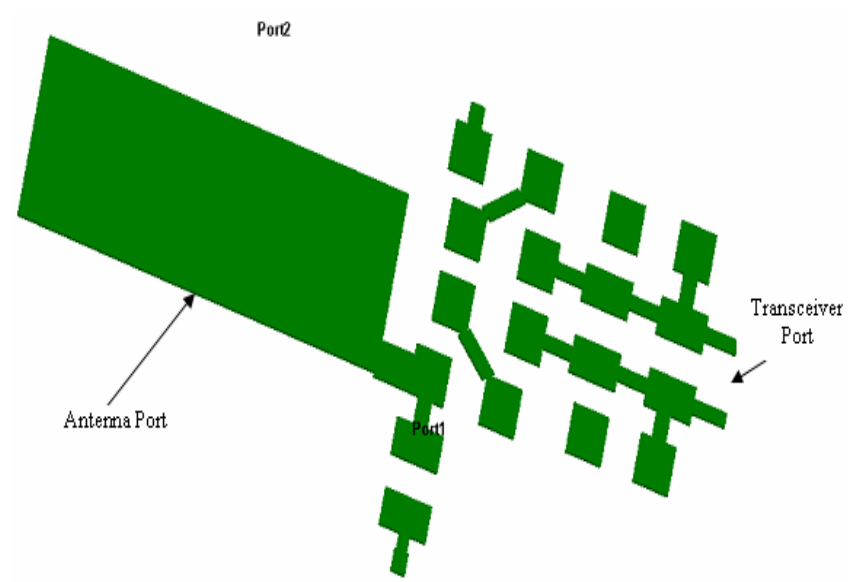

Figure 7. Matching network Ansoft planer model

From Figure. 8 it can be seen that the optimization process results in a significant improvement in return loss through tuning one of the matching network inductors of initial value $12 \mathrm{nH}$.

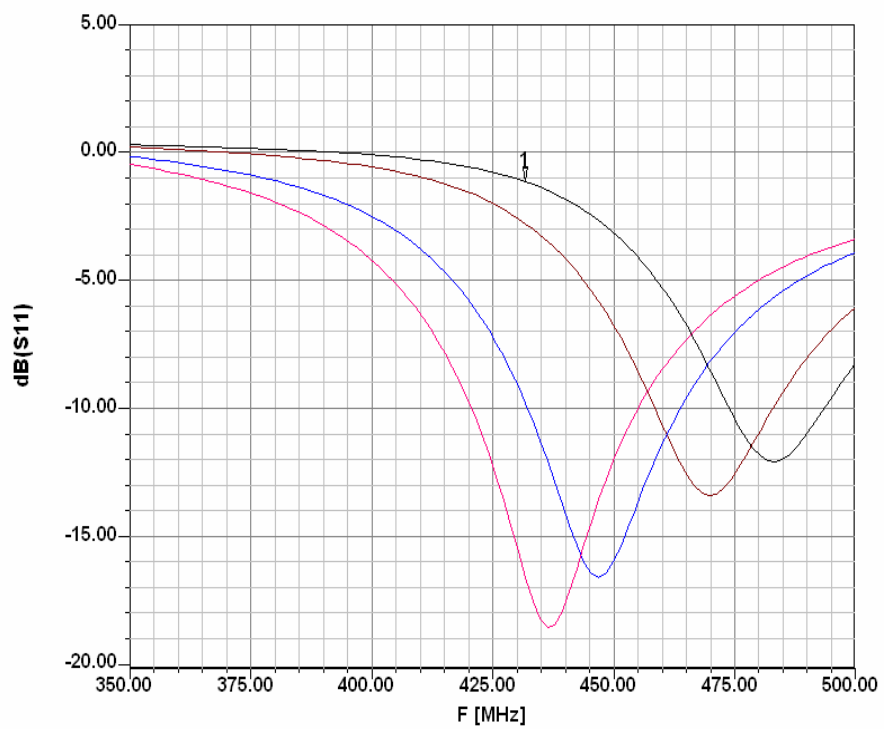

Figure 8. Simulated Return Loss using the Optimization process

\section{B. Antenna Design}

Because of the high space restrictions of the application, a special miniaturized $50 \Omega$ chip antenna of size $16 \times 3 \mathrm{~mm}$ has been used. A single ended matching network with values identified by the described optimization process was adopted between the antenna and transceiver. The antenna has been placed carefully on the PCB where few factors like the ground plane, size of the feeding line antenna directivity and enough clearance have been taken first into consideration. To have a closer look on how the antenna is impedance 
matched to RF circuit, the network analyzer 8510 from Hewlett Packard has been employed for this purpose. Figure 9 shows the measured return power patterns for the RF circuit in a narrow band $(\sim 70 \mathrm{MHz})$.

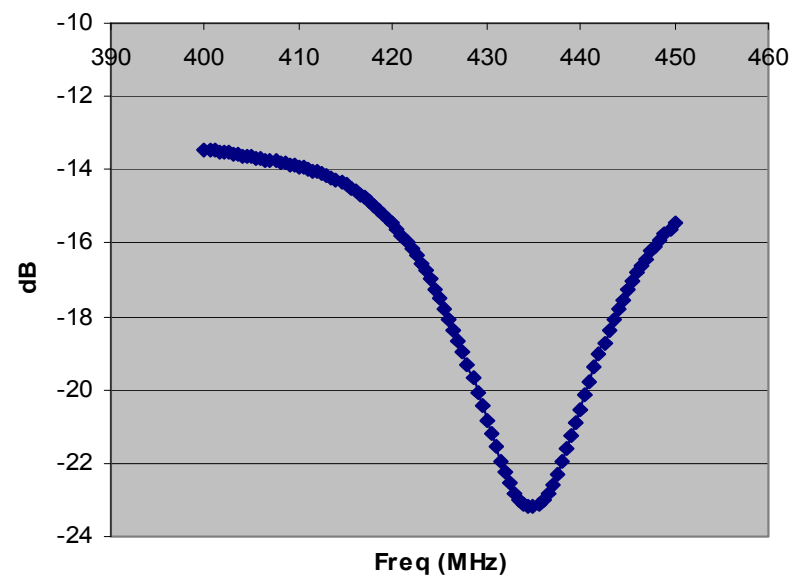

Figure 9. Measured return power loss from the RF transceiver

It is obvious that RF unit displays a good power response at the desired frequency and confirms the results obtained from the Ansoft simulation.

\section{EXPERIMENTAL RESULTS}

The telemetric link and its capabilities to send information have been examined in a building environment. A pressure range from 0 to $10 \mathrm{kPa}$ has been generated artificially and applied to examine the sensor response. Figure 10 shows a sample result for the sensor sensitivity against pressure.

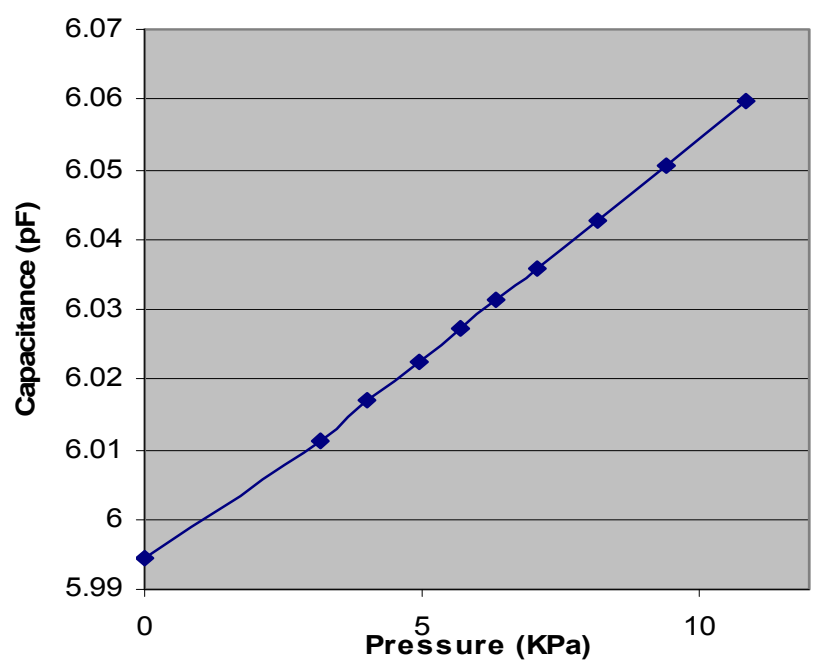

Figure 10. Change in capacitiace with pressure in the range $0-10 \mathrm{kPa}$

\section{CONCLUSIONS}

This work describes the development of a novel wireless sensor prototype suitable for capacitive sensors monitoring in building environment. The pressure sensors are fabricated based on the heavily doped compensated SiGeB.

The paper outlined a procedure to design and optimize the RF matching circuit on a PCB substrate. The methodology allowed the optimization of the ISM wireless node with good correlation having been achieved between simulation and measurement. This analysis helps ensure that matching circuits such as this can be optimized predictably, prior to building any hardware and thus avoiding the need for extensive lab testing or numerous and costly PCB re-spins

The RF antenna part with the impedance matching circuit has been designed with great attention. Obviously the RF circuit showed a superior level of power reflected back $(<-$ $22 \mathrm{~dB}$ ). The SiGeB pressure sensors were evaluated using the wireless prototype imposed to a certain pressure range. It was seen that sensors displayed a high sensitivity to the pressure changes in the range $0-10 \mathrm{kPa}$ and such performance it expected even beyond this range.

\section{ACKNOWLEDGMENT}

We would like to thank Tyndall National Institute, Microelectronics Applications Integration (MAI) for their support and technical advice.

\section{REFERENCES}

[1] A. Leung,W. H.Ko, T.M. Spear, and J. A. Bettice, "Intracranial pressure telemetry system using semicustom integrated circuits," IEEE Trans.Biomed. Eng., vol. 33, pp. 386-395, Apr. 1986.

[2] T. Kim, T. Kim, Y.-G. Ha, J. Kang, D. A. K. D. Kim, C. P. A. K. C. P. Kit, and J.-C. A. S. J.-C. Sohn, "Experiments on Building Ubiquitous Robotic Space for Mobile Robot Using Wireless Sensor Networks " presented at 22nd International Conference on Advanced Information Networking and Applications - Workshops, 2008.

[3] J.W. Fonda, S.E. Watkins, S. Jagannathan, and M. Zawodniok, "Embeddable sensor mote for structural monitoring," Proceedings of SPIE - The International Society for Optical EngineeringK, vol.6932, 2008

[4] A.M. Suva, A. Correia, A.J. Gano, A.M. de Campos, I. Teixeira, "Wireless intelligent sensor modules for home monitoring and control," IEEE International Workshop on Intelligent Signal Processing, pp.110-115, 2005.

[5] http://www.ansoft.com/products/hf/ansoft_designer/

[6] J. Silvestro, J. Silvestro, J. DeLap, and T. Donisi, "Antenna and system design with Ansoft Antenna and system design with Ansoft," presented at IEEE International Symposium on Phased Array Systems and Technology, 2003. 